\title{
U0126 inhibits pancreatic cancer progression via the KRAS signaling pathway in a zebrafish xenotransplantation model
}

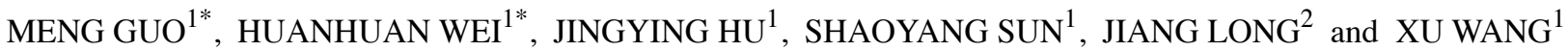 \\ ${ }^{1}$ Key Laboratory of Metabolism and Molecular Medicine, Ministry of Education, Department of Biochemistry \\ and Molecular Biology, School of Basic Medical Sciences, Fudan University; ${ }^{2}$ Department of Pancreatic \\ and Hepatobiliary Surgery, Fudan University Shanghai Cancer Center, Shanghai 200032, P.R. China
}

Received March 20, 2015; Accepted April 24, 2015

DOI: $10.3892 /$ or.2015.4019

\begin{abstract}
Pancreatic cancer is one of the most aggressive human cancers, and the pharmaceutical outcomes for its treatment remain disappointing. Proper animal models will provide an efficient platform for investigating novel drugs, and the zebrafish has become one of the most promising and comprehensive model animal in cancer research. In the present study, we used a novel xenograft model in zebrafish by transplanting human pancreatic cancer cells to study the progression and metastasis of pancreatic cancer cells and to assay the pharmacological effects of new drug U0126 in vivo. We first established a primary xenograft model of pancreatic cancer by injecting human pancreatic cancer cells into both live larval and adult zebrafish, and then investigated the behaviors of CM-DiI-labeled human pancreatic cancer cells. Subsequently, we tested the potential of this model for drug screening by evaluating a known small-molecule inhibitor, U0126, which targets the KRAS signaling pathway. Cells with KRAS mutations exhibited significant proliferative and migratory behaviors and invaded the zebrafish vasculature system. In contrast, the proliferation and migration of Mia PaCa-2 cells in zebrafish larvae were substantially repressed following U0126 treatment. These results suggest that zebrafish xenotransplantation can be used as a simple and efficient tool to screen and identify new anti-pancreatic cancer compounds.
\end{abstract}

Correspondence to: Professor Xu Wang, Key Laboratory of Metabolism and Molecular Medicine, Ministry of Education, Department of Biochemistry and Molecular Biology, School of Basic Medical Sciences, Fudan University, 138 Yixueyuan Road, Shanghai 200032, P.R. China

E-mail: wangxu2013@fudan.edu.cn

Professor Jiang Long, Department of Pancreatic and Hepatobiliary Surgery, Fudan University Shanghai Cancer Center, 270 DongAn Road, Shanghai 200032, P.R. China

E-mail: surgeonlong@fudan.edu.cn

${ }^{*}$ Contributed equally

Key words: xenograft model, zebrafish, pancreatic cancer, KRAS signaling pathway, small molecule inhibitor, drug screening

\section{Introduction}

Pancreatic cancer is one of the most aggressive malignancies worldwide, with a median survival time of less than 6 months and with a 5-year overall survival rate less than $5 \%$ (1). The annual death rate for pancreatic cancer is almost equal to the incidence rates in both developed and developing countries, suggesting the lack of an effective screening method for targeted drug therapy (2-4). Since traditional drug development processes are slow and expensive, newer and more effective high-throughput animal models are greatly needed to promote preclinical therapies for pancreatic cancer (5). Zebrafish models have been shown to be effective drug discovery tools due to the following characteristics: high fecundity, small size, feasible manipulation, easy to observe, rapid development and analogous structures to other vertebrates (6). In addition, the zebrafish at larval stages is transparent with an immature immune system, making it an ideal model for cancer xenotransplantation and in vivo fluorescent imaging. The efficiency of drugs in inhibiting tumors can be observed at the cellular level in vivo and assessed precisely at the whole organ level $(7,8)$. Xenotransplantation studies in zebrafish are potentially valuable for investigating pancreatic cancer biology, particularly for assaying high-throughput screening drugs in vivo (9). In the present study, we performed xenotransplantation of tumor cell lines derived from pancreatic cancer in both larval and adult zebrafish and demonstrated the advantages of the zebrafish as an in vivo model by which to test potential drug therapies for pancreatic cancer.

Activating mutations in the KRAS signaling pathway have been observed in $90 \%$ of pancreatic cancer patient tissues, indicating a major role for these mutations in pancreatic cancer initiation and progression $(10,11)$. Aberrant activation of RAS-mediated proliferation and survival signaling pathways in cells can be initiated by activating mutations in KRAS. Targeting KRAS mutations may reduce cancer mortality; nevertheless, effectively targeting of KRAS has proven challenging for clinical treatments thus far (12). Inhibiting the downstream effector MEK1/2, a dual-specific kinase that is required for ERK1/2 activation and that is sustained at a high level through the Raf/MEK/ERK pathway driven by mutant KRAS, has been shown to be effective in preclinical studies (13). Multiple small-molecule inhibitors of MEK are 
now being studied in preclinical or clinical trials to treat malignant neoplasms (14). Identifying and characterizing druggable inhibitors of crucial downstream effectors of KRAS have also been challenging, suggesting that new animal models for drug screening in vivo are required. The compound U0126 was identified as an inhibitor that directly suppresses MEK $1 / 2$ activation $(15,16)$. Here, we used U0126 as an example and present a robust in vivo system for evaluating the effectiveness of a suppressor molecule.

First, we investigated whether xenografted Mia PaCa-2 (human pancreatic cancer) cells could proliferate and spread in vivo while retaining the biological features of carcinoma in zebrafish. Then, we attempted to verify and quantify the proliferation and migration of fluorescence-labeled pancreatic cancer cells in vivo by imaging analysis. Additionally, we studied the interaction between pancreatic cancer cell dispersal and angiogenesis. Finally, we investigated whether U0126 suppressed the proliferation of Mia PaCa- 2 cells in the xenotransplanted zebrafish larvae and compared those results with our in vitro experiments. Our findings indicated that the zebrafish can be utilized as a rapid and simple animal model that can be visualized for cancer metastasis mechanistic research and anti-pancreatic cancer molecular inhibitor screening.

\section{Materials and methods}

Cell culture and proliferation assay. The pancreatic cancer cell lines Mia PaCa-2 and BxPC-3 were purchased from the American Type Culture Collection (ATCC; Rockville, MD, USA) and grown in Dulbecco's modified Eagle's medium (DMEM) and high-glucose RPMI-1640 (Invitrogen Life Technologies, Carlsbad, CA, USA) supplemented with $10 \%$ fetal bovine serum (FBS) and $1 \%$ penicillin-streptomycin at $37^{\circ} \mathrm{C}$ in $5 \% \mathrm{CO}_{2}$. A final concentration of $5 \mu \mathrm{M}$ CM-DiI (Invitrogen Life Technologies) was added to PBS-washed cells and incubated for $15 \mathrm{~min}$ in a $\mathrm{CO}_{2}$ incubator at $37^{\circ} \mathrm{C}$ followed by another 15 -min incubation at $4^{\circ} \mathrm{C}$. The cells were pelleted, washed, and resuspended in DMEM before transplantation.

Mia PaCa-2 cells were seeded at 5,000 cells/well in 96-well plates and allowed to stabilize in DMEM complete media. After $24 \mathrm{~h}$, the cells were treated with $0,2.5,5,10,20$, 40 or $80 \mu \mathrm{M}$ U0126 in DMEM media supplemented with $0.5 \%$ FBS. The cells were maintained for $12 \mathrm{~h}$, incubated with $10 \%$ 3-(4,5-dimethylthiazol-2-yl)-2,5-diphenyltetrazolium bromide (MTT; Sigma-Aldrich, St. Louis, MO, USA) for $4 \mathrm{~h}$, aspirated and dissolved in DMSO to generate the formazan product. Absorbance was measured at $560 \mathrm{~nm}$ with the reference wavelength at $700 \mathrm{~nm}$.

Zebrafish and pancreatic cancer transplantation. Larval and adult zebrafish were obtained, staged and raised from natural spawnings of wild-type (AB) and $T g(f k l: E G F P)$ zebrafish [visualization of vascular endothelium (18)]. All the zebrafish utilized in the subsequent experiments were maintained in accordance with the Molecular Medicine Laboratory of Fudan University guidelines. Zebrafish embryos were obtained using standard mating conditions and were de-chorionized at $48 \mathrm{~h}$ post-fertilization (hpf).
For transplantation of the larvae, the embryos were anesthetized with $0.003 \%$ tricaine and positioned with their right side up on a wet agarose pad. Cells were acquired from culture dishes using a non-enzymatic cell-lifting solution and resuspended in PBS at a concentration of $10^{6}$ cells $/ 50 \mu \mathrm{l}$ and maintained on ice before injection. Approximately 100-200 cancer cells were injected into the perivitelline cavity of each zebrafish larva using an Eppendorf FemtoJet injector equipped with a $0.75-\mathrm{mm}$ borosilicate glass needle. The injection parameters were as follows: injection pressure, $20 \mathrm{hp}$; holding pressure; and injection time, $30 \mathrm{sec}$. Immediately following transplantation, the injected larvae were washed and selected under a fluorescence-stereo microscope. The larvae with fluorescence-positive cells were separated into individual containers and kept at $35.5^{\circ} \mathrm{C}$.

For adult transplantation, the 6-mpf zebrafish were irradiated with 25 Gy of $\gamma$-irradiation at 2 days before transplantation and allowed to recover in standard fish water. On the day of the transplant, recipients were anesthetized in $0.2 \%$ tricaine. Using a Hamilton syringe, $10 \mu 1$ of cell suspension was transplanted into the cardiac chamber, and the fish was immediately returned to fresh fish water. Recipients were kept off of system water (to minimize infectious risk) for 1 week and fed standard flake/brine shrimp meal.

All animal procedures were approved by the Institutional Animal Care Committee, Fudan University, China.

Histological analysis. Adult fish were fixed in $4 \%$ paraformaldehyde overnight. After the fish were processed and embedded in paraffin, 5-mm sections were cut either longitudinally or transversely. Staining was performed with hematoxylin and eosin.

Morphological analyses and drug treatment. Transplanted zebrafish were subsequently visually documented on a daily basis. Live zebrafish embryos and adults were anesthetized with tricaine before microscopic observation. Digital micrographs were acquired using a DP70 CCD camera mounted on an IX71 inverted fluorescence microscope (both from Olympus, Japan). Two images were acquired in the same focal plane in bright field and in transmitted light passing through the RFP or GFP filters and then superimposed and processed using the Adobe Photoshop program. In processing the fluorescent images, the RFP signal in the zebrafish embryos changed dynamically. Image-Pro Plus 6.0 (Media Cybernetics, Rockville, MD, USA) software was used to analyze the imaging data after transplantation and to quantify the fluorescence variation that represented the dynamic changes in the cell counts and locations. Images that were more sophisticated were obtained using light sheet microscopy (Zeiss, Germany).

For drug treatment, U0126 (Cell Signaling Technology, Beverly, MA, USA) was dissolved in DMSO to $1,000 \mu \mathrm{M}$ and added directly to the water at a final concentration of $4 \mu \mathrm{M}$. Equivalent DMSO was used as a vehicle control. E3 medium plus drug was changed every $24 \mathrm{~h}$.

Western blot analysis. Total proteins were separated by SDS-PAGE and transferred to membranes. After blocking with milk, the membranes were incubated in a milk solution containing phospho-p44/42 MAPK (Erk1/2) rabbit mAb and 
A

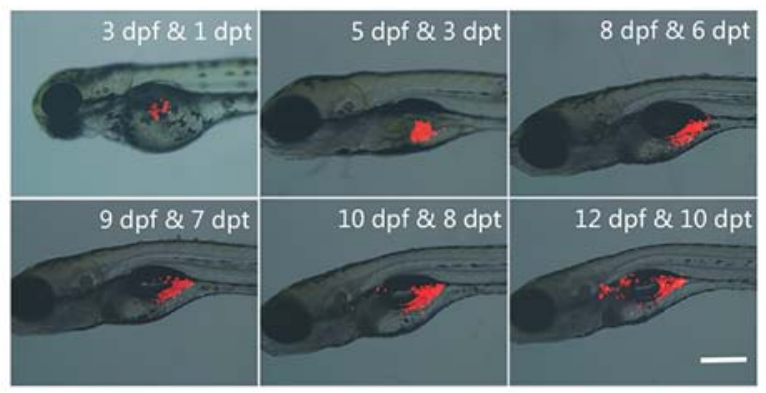

C

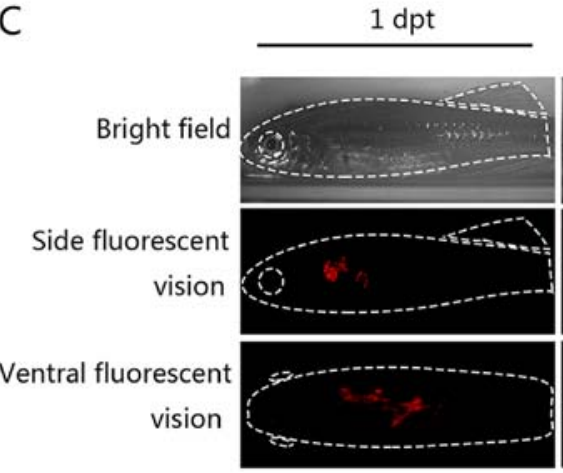

D

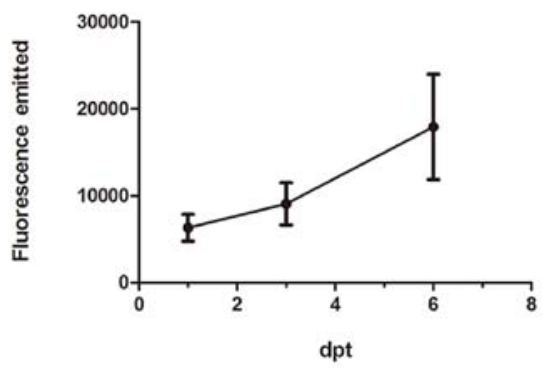

B

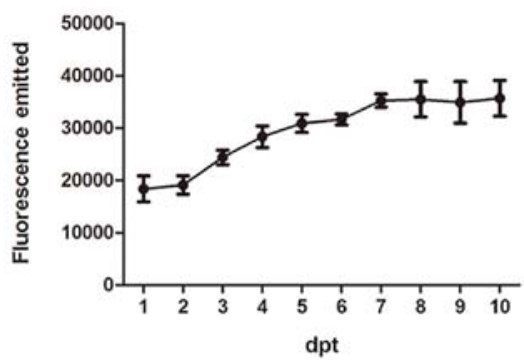

$d p t$

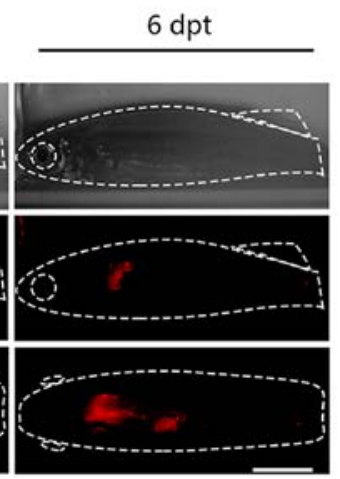

E

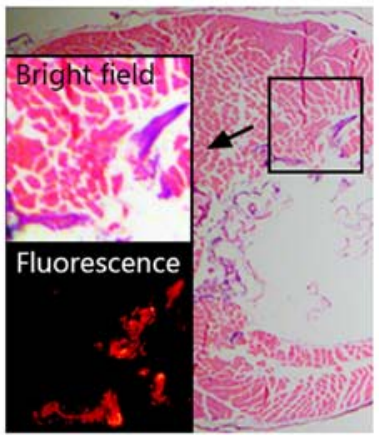

Figure 1. The transplanted human pancreatic cancer cells survive and proliferate in larval and adult zebrafish. (A) Mia PaCa-2 pancreatic cancer cells survived in the zebrafish larvae body, were concentrated in clusters at $3 \mathrm{dpt}$, disseminated in the vitelline sac by $6 \mathrm{dpt}$ and were transferred into the enterocoelia and pancreas at $10 \mathrm{dpt}$. Scale bar, $50 \mu \mathrm{m}$. (B) Counting the cell numbers daily in larvae revealed Mia PaCa- 2 cell proliferation. Quantitative values are means \pm SEM from 20 independent individuals. (C) Red Mia PaCa-2 cells proliferated and dispersed in adult fish within 7 days after xenotransplantation. Scale bar, 3 mm. (D) These results mirrored the fluorescence imaging assays and were consistent with the histological results. Quantitative values are means \pm SEM from 7 independent individuals. (E) The histological characteristics of Mia PaCa-2 cells invading the digestive organs and abdominal wall are clearly observed (magnification, x200). High-magnification image (x400) of the black box is shown in the upper-left image, and the same result is shown in the fluorescence image (lower-left). dpt, days post-transplantation.

p44/42 MAPK (Erk1/2) rabbit mAb (both from Cell Signaling Technology). The bound antigen-antibody complex was detected by a secondary antibody (Cell Signaling Technology) using a chemiluminescence kit (Millipore, Germany). The same membrane was used to determine $\beta$-actin levels, which were detected using an anti- $\beta$-actin antibody (Sigma-Aldrich), as an internal loading control.

KRAS mutation analysis. Mutation detection was performed by direct DNA sequencing of kras exons 2 and 3 as described previously (17).

Statistical analysis. The dynamic changes in the pancreatic cancer cells, as represented by fluorescence variations counted by Image Pro Plus 6.0 analyses, were compared between the drug-treated and control animals. Fisher's exact test was performed in SAS 9.1 (SAS Institute, Cary, NC, USA) to determine P-values. P-values $<0.05$ were considered to indicate statistically significant results.

\section{Results}

Xenografted human pancreatic cancer cells were able to proliferate and migrate in zebrafish larvae and adults. First, we conducted a comparison and analysis of two human pancreatic cancer cell lines, Mia PaCa-2 and BxPC-3, to select an appropriate source for xenografting. After the xenografts were performed, we observed that the Mia PaCa- 2 and $\mathrm{BxPC}-3$ cells proliferated and disseminated throughout the zebrafish bodies intravitally at $6 \mathrm{~h}$ post-transplantation (hpt). We microinjected CM-DiI-labeled Mia PaCa-2 cells into the perivitelline cavity of $48 \mathrm{hpf}$ embryos and analyzed the larvae within 10 days 


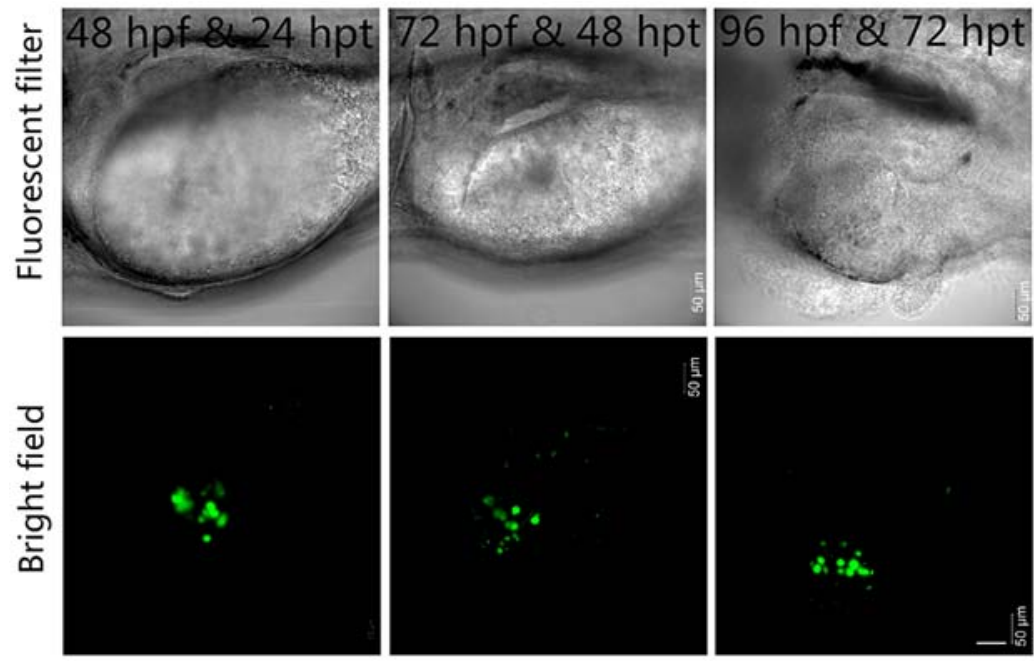

Figure 2. GFP-labeled BxPC-3 cells showed no metastasis and proliferation within $72 \mathrm{hpt}$ in vivo. Scale bar, $500 \mu \mathrm{m}$. hpt, hours post-transplantation.
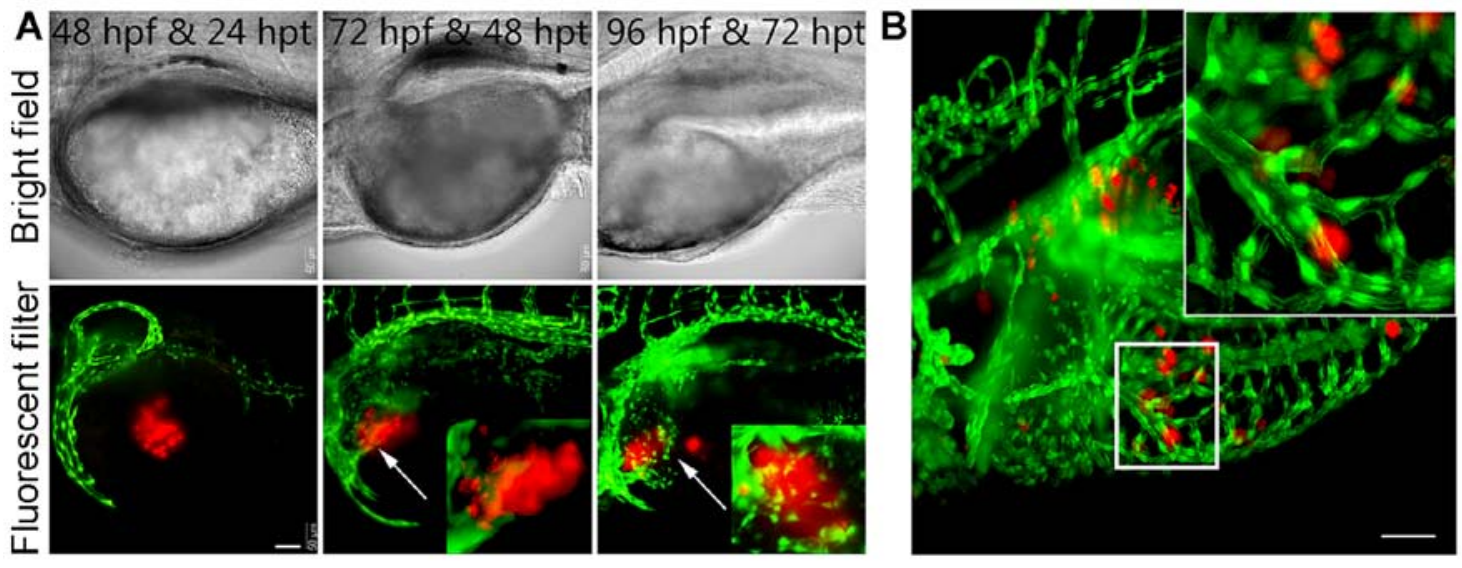

Figure 3. The transplanted human pancreatic cancer cells invade zebrafish vasculature. (A) Red human cancer Mia PaCa-2 cells disseminated in the $T g(f k l: E G F P)$ transgenic zebrafish larvae and spread throughout the developmental vasculature from 24 to $72 \mathrm{hpt}$. The arrowhead-labeled regions at a higher magnification are shown (lower-right). Scale bar, $50 \mu \mathrm{m}$. (B) Transplanted Mia PaCa-2 cells were transferred to the larval vasculature and induced the vasculature to approach the cell mass. The white box at a higher magnification is shown in the upper-right image. Scale bar, $50 \mu \mathrm{m}$. hpt, hours post-transplantation.

by continuous fluorescence microscopy. We noted that the majority of larvae (86\%) showed tumor cells within their bodies after a 12-h period and that the cells were concentrated at $36 \mathrm{hpt}$ (Fig. 1A). Moreover, we detected xenografted cells that appeared to disseminate in the vitelline sac at 6 days post-transplantation (dpt) and to spread into the body cavity and pancreas until $10 \mathrm{dpt}$ (Fig. 1A). In addition, we confirmed CM-DiI-labeled Mia PaCa-2 cell proliferation by recording and analyzing the images daily (Fig. 1B). In contrast, we found that EGFP-labeled BxPC-3 cells barely proliferated and disseminated outside the yolk sac region (Fig. 2). Therefore, we utilized Mia PaCa-2 cells as the grafted cells in subsequent transplantation experiments.

To assess the broad distribution of the engrafted cells in zebrafish tissues, we next investigated the morphological characteristics of the CM-DiI-labeled Mia PaCa- 2 cells after xenografting in adult fish and performed histological analysis using hematoxylin and eosin staining. We determined that the fluorescently labeled Mia $\mathrm{PaCa}-2$ cells were able to proliferate and migrate to the pancreas within 7 days after intraperitoneal xenotransplantation into the irradiated adult fish (Fig. 1C).
Additionally, Mia PaCa-2 cell proliferation in the adult fish was consistent with the observations made in the juvenile fish (Fig. 1D).

To further verify the previous results, we prepared serial tissue slices and then visualized the hematoxylin and eosinstained images under fluorescence. Histological analysis revealed that the $\mathrm{Mia} \mathrm{PaCa}-2$ cells were distributed in the digestive organs and abdominal wall (Fig. 1E). The fluorescence persisted in the adjacent serial histological sections, demonstrating the existence of human pancreatic cancer cells in accordance with the histological analysis (Fig. 1E, lowerleft inset image). These results suggest that the Mia $\mathrm{PaCa}-2$ human pancreatic cancer cell line is competent for use in zebrafish xenografting. Furthermore, the proliferation of the xenografted human pancreatic cancer cells was able to be visualized in the living zebrafish.

Pancreatic cancer cells spread and migrate throughout the vasculature in zebrafish larvae. To study the initial tumor formation by the transplanted human pancreatic cancer cells in vivo, we investigated the morphological characteristics of 
A

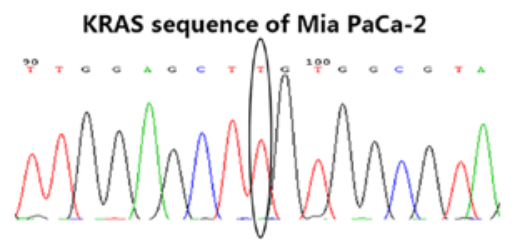

KRAS sequence of BXPC-3

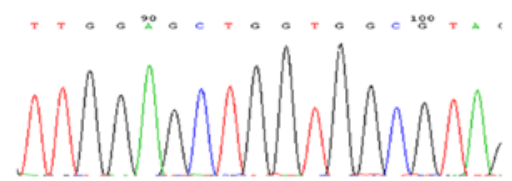

C

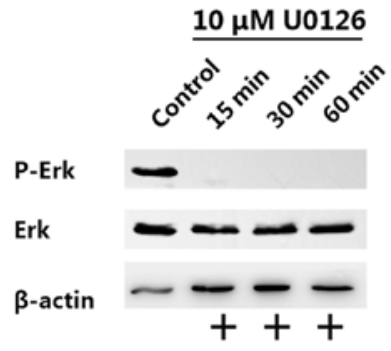

[)

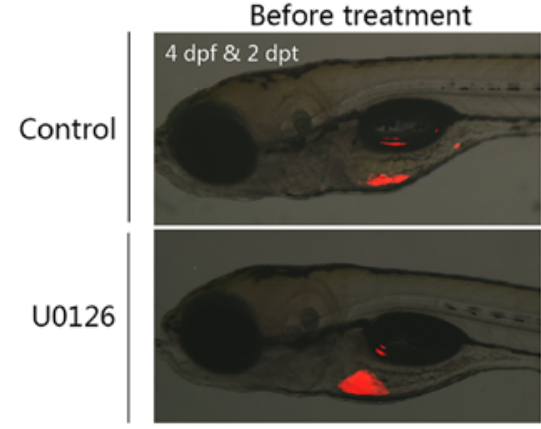

E

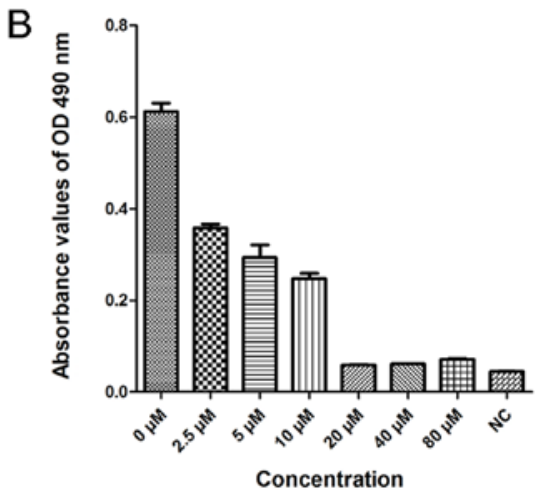

U0126 treated for $60 \mathrm{~min}$

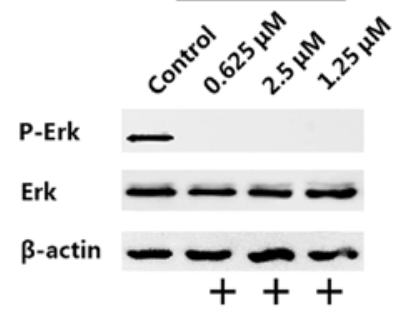

2 days after treatment

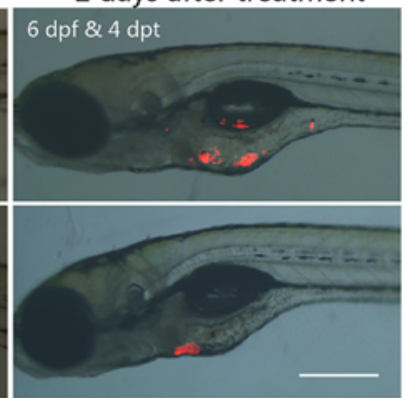

$96 \mathrm{hpt}$

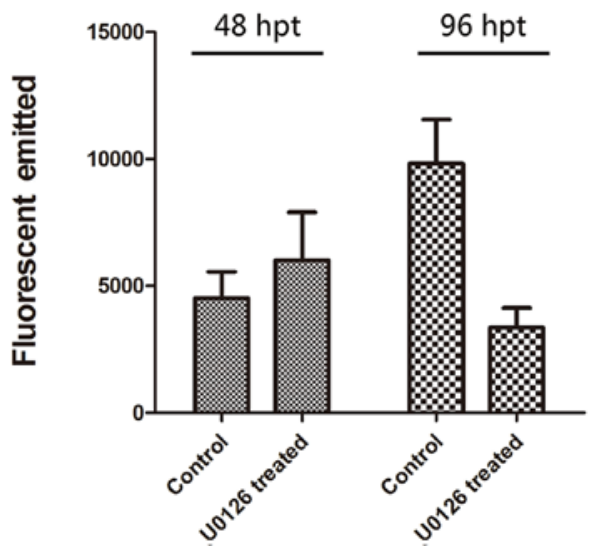

Figure 4. U0126 treatment suppresses human pancreatic cancer cell proliferation in vitro and in zebrafish in vivo via inhibition of MEK phosphorylation. (A) Mia PaCa-2 cells have a missense mutation at kras codon 12 and BxPC-3 cells express the wild-type. (B) MTT analysis demonstrated that U0126 inhibited Mia PaCa-2 cell proliferation in vitro. Quantitative values are means \pm SEM from 3 independent experiments. (C) Western blot analysis of ERK phosphorylation confirmed the suppression of MEK downstream pathways. (D) The proliferation and metastasis of xenografted Mia PaCa-2 cells were suppressed following treatment with U0126, while control animals showed marked proliferation and metastasis. Scale bar, $30 \mu \mathrm{m}$. (E) Compared with the control group, the reduced levels of proliferation were statistically significant $(\mathrm{P}<0.05)$ after exposure to U0126. Quantitative values are means \pm SEM from 60 independent individuals for each group. dpt, days post-transplantation; hpt, hours post-transplantation.

these cells by confocal laser scanning microscopy during the preliminary stage of transplantation. Although transplanted Mia PaCa- 2 cells can barely form solid tumor masses that resemble the ones shown within human patients and mammalian xenograft models, we identified several CM-DiI-labeled
Mia PaCa- 2 cell clusters that were integrated into the intestinal region (Fig. 3).

The later steps of invasive cancer cell progression involve locating vessels, invading and inducing angiogenesis, which facilitates the extravasation of the cells to form secondary 


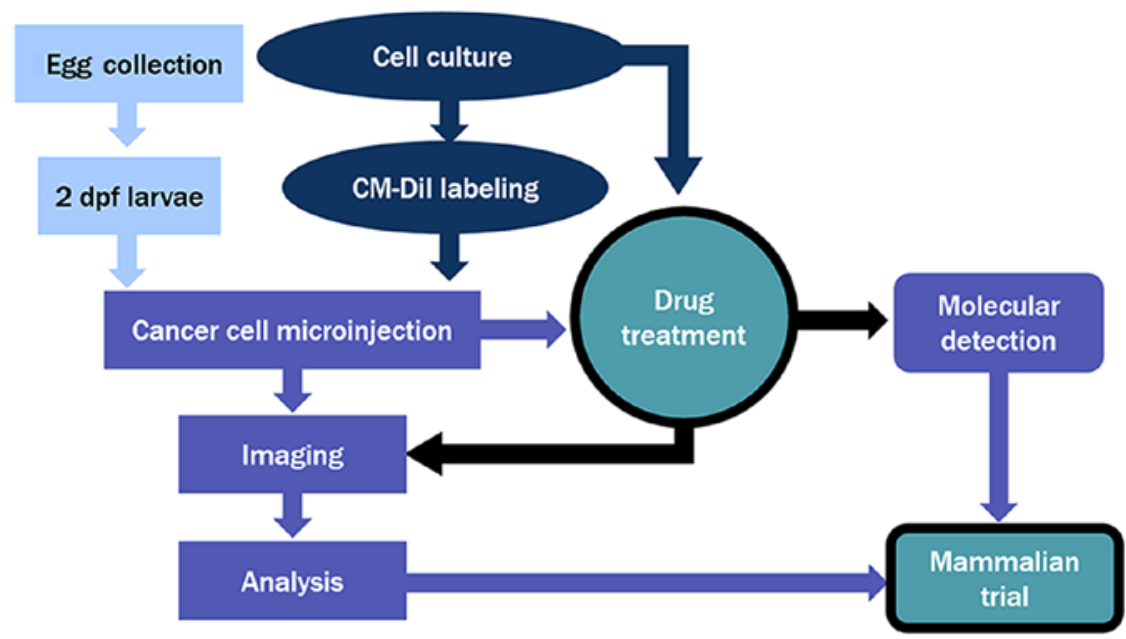

Figure 5. The zebrafish xenotransplantation model as a platform for drug development. dpt, days post-transplantation.

tumors $(18,19)$. To investigate the process of pancreatic cancer cell invasion of the vascular system of zebrafish and induction of angiogenesis, we performed microinjections of CM-DiI-labeled Mia PcCa-2 cells into the yolk and primordial cavity of $T g(f l k l: E G F P)$ transgenic zebrafish larvae at $2 \mathrm{dpf}(18)$. The human pancreatic cancer cluster was initially distinct from the developing vasculature of the larvae (Fig. 3A, left image). Over time, however, the xenografted Mia PaCa-2 cells had a tendency to migrate to the vasculature (Fig. 3A, middle image), and the developing zebrafish vasculature extended toward and directly contacted the tumor clusters (Fig. 3A, middle and right images). These results indicated that the human pancreatic cancer cells stimulated angiogenesis and recruited blood vessels from the host larvae that subsequently supported the continued growth of the tumor.

To study the effect of the developing vascular distribution on the diffusion of the human pancreatic cancer cells, we further observed the advanced aspects of xenotransplanted zebrafish. Approximately $30 \%$ of the xenografted larvae exhibited Mia PaCa- 2 cell clusters that diffused within the invading blood vessels and that spread throughout the vasculature (Fig. 3B). Therefore, all of these results corroborated the findings that pancreatic cancer cells were able to spread throughout the vasculature and stimulate angiogenesis in the zebrafish.

Treatment with U0126 deregulates KRAS activity in human pancreatic cancer cells in live zebrafish. To investigate whether larval zebrafish are suitable for in vivo drug screening, we tested the effects of a targeted inhibitor of KRAS signaling on xenotransplanted human pancreatic cancer cells in our zebrafish model. The compound U0126 is able to directly suppress MEK1/2 activation, which is critical for transmitting signals to ERK (15). This effector can be abnormally sustained in an activated state by mutated KRAS signaling and is thus an attractive pharmaceutical target for tumors harboring aberrant MAPK pathway signaling $(11,12,20)$.

First, we performed kras gene sequencing in the two cell lines to investigate the inhibitory effect of U0126 on human pancreatic cancer cells with KRAS mutations. Our sequencing results showed that BxPC-3 cells expressed the wild-type kras gene, whereas Mia PaCa- 2 cells had a missense mutation at codon 12 (Fig. 4A). In addition, we verified that U0126 exerted an inhibitory effect on $\mathrm{Mia} \mathrm{PaCa}-2$ cell proliferation in vitro. U0126 treatment lead to dose-dependent suppression of cell proliferation at concentrations ranging from 2.5 to $80 \mu \mathrm{M}$, and the maximum inhibitory concentration was $20 \mu \mathrm{M}$ (Fig. 4B).

To validate the U0126-induced inhibition of MEK1/2 phosphorylation to suppress mutated KRAS signaling, we tested the time- and dosage-effect relations after treating $\mathrm{Mia} \mathrm{PaCa}-2$ cells with U0126 and then investigated ERK phosphorylation. Western blot analysis showed that MEK phosphorylation in Mia $\mathrm{PaCa}-2$ cells was inhibited by U0126 treatment after a short incubation period (15 min) and at low concentrations (0.625 $\mu \mathrm{M})$ (Fig. 4C). MEK phosphorylation is critical to the activation of the primary route of signal transmission in the MAPK pathway, and MEK phosphorylation is inhibited by U0126 (21). These results suggest that U0126 can suppress Mia $\mathrm{PaCa}-2$ cell proliferation by inhibiting MEK phosphorylation.

Next, we assessed the efficacy of U0126 to validate our zebrafish xenograft model system. As demonstrated in the above experiments, U0126 inhibited cell proliferation in vitro in a dose-dependent manner, and the inhibitory concentration was used as the basis of the $\mathrm{IC}_{50}$ value calculation. In our xenograft model, U0126 produced a prompt effect that suppressed cell proliferation (Fig. 4D). We exposed xenotransplanted larvae to U0126 for $48 \mathrm{~h}$ at a final concentration of $4 \mu \mathrm{M}$ in an aqueous medium. The xenotransplanted larvae in the experimental group exhibited $29.28 \pm 9.2 \%$ cancer cell degeneration following U0126 treatment in 60.0\% (36 in 60) of the larvae. In contrast, the corresponding control group (untreated) showed $121.05 \pm 37.2 \%$ cancer cell proliferation in $85.0 \%$ (51 in 60) of the larvae. Representative images acquired before and after processing are shown in Fig. 4D. Moreover, statistical analysis showed that U0126 significantly inhibited Mia PaCa-2 cell proliferation compared with the in vivo results $(\mathrm{P}<0.05$, Fig. $4 \mathrm{E})$. In addition, we detected fewer metastases in the bodies of the larvae in the experimental group; however, the mock-treatment group displayed frequent 
metastasis. These assays indicated that U0126 can suppress the proliferation and metastasis of pancreatic cancer cells by inhibiting KRAS signaling downstream effector activation both in vivo and in vitro, suggesting that this xenotransplantation pancreatic cancer model utilizing larval zebrafish is useful for facilitating in vivo drug screening.

\section{Discussion}

The development of a proper animal model test will provide an efficient platform for investigating novel drugs. Utilizing xenograft models that recapitulate key processes of cancer development may be extremely favorable for screening large numbers of small-molecule compounds in an in vivo setting. Presently, several targeted therapeutic strategies have been used in pancreatic cancer preclinical studies (22). Pancreatic cancer genetic alterations in the KRAS signaling pathway are involved in over $90 \%$ of human pancreatic cancer cases. The components in this pathway are promising targets for identifying novel therapies (23). The most common KRAS mutation in human pancreatic cancer is a gain-of-function mutation at codon 12. Moreover, pancreatic cancer cell growth has been shown to be dependent on the activity of the mutated kras gene; accordingly, silencing the kras gene has been shown to be effective in controlling pancreatic cancer cell line proliferation (24). However, the clinical development of targeted agents for pancreatic cancer has not been successful. Most known inhibitors have already been processed preclinically and represent promising targets for therapeutic intervention; however, these inhibitors lack concise and effective in vivo studies (11). In the present study, we harnessed the advantages of xenograft models of pancreatic cancer in zebrafish for KRAS signaling pathway inhibitor screening.

The zebrafish model offers a rapid and inexpensive platform with which to investigate the proliferation and metastatic potential of human cancer cells. Moreover, the larvae experimentation allowed us to intuitively evaluate the mechanism by which U0126 targets tumor cell signaling pathways. In the present study, we described this zebrafish xenograft model, which provides an excellent platform to study human pancreatic cancer cell proliferation and migration and the relationship with angiogenesis in vivo. The study indicated that Mia PaCa-2 cells with kras codon 12 mutation showed stronger proliferative and metastatic ability than BxPC-3 cells in vivo (Figs. 1, 2 and 4A). These result suggest that xenotransplantation of cells with KRAS mutation was able to simulate pancreatic cancer initiation and progression in vivo.

In the present study, we utilized the KRAS signaling inhibitor U0126 as an example to test the feasibility of this model for high-throughput screening. Although the pharmacological characteristics of U0126 indicated that this drug was unsuitable for clinical development, the small-molecule compound was ultimately demonstrated to be an efficient inhibitor when used in multiple studies $(13,25)$. We first described the inhibitory role of U0126 in suppressing Mia $\mathrm{PaCa}-2$ cell proliferation in vitro due to the KRAS activating mutation in these cells. In addition, we tested ERK phosphorylation following U0126 treatment to verify that U0126 could target the downstream effectors of KRAS signaling. Finally, we validated our findings that the proliferation of transplanted Mia PaCa- 2 cells could be inhibited by exposure to U0126, which was consistent with the findings of another study (26). The image analysis combined with the statistical results also showed that U0126 markedly suppressed cell growth in the majority of transplanted individuals. All these results demonstrated that this elementary and reliable xenotransplantation model in zebrafish is effective for investigating anti-pancreatic cancer drugs.

Metastasis is the major cause of death in pancreatic cancer patients and is not fully understood. An intriguing observation in our study was the phase relationship between the transfer of pancreatic cancer cells and the vascular development of larvae. We dynamically detected that xenografted Mia PaCa-2 cells migrated to the host vascular system, induced angiogenesis and diffused through developing vessels in the $\operatorname{Tg}(f k 1$ :EGFP) larvae. Neovascularization could offer the necessary material for tumor metabolism (27), although these components are not yet clear. In contrast, xenotransplanted Mia PaCa- 2 cells may recruit new blood vessels; this recruitment is one of the typical forms of hematogenous metastasis. Therefore, we propose that the zebrafish xenograft model may allow us to investigate hematogenous pancreatic cancer cell metastasis and tumorinduced angiogenesis in vivo.

Transplantation of primary cancer cells from tissue culture could offer a more realistic model than cell lines. All of our analyses were performed using human pancreatic cancer cell lines, which confirmed the use of CM-DiI labeling as an efficient and feasible technique (28). Primary cancer cells can also be tagged through this method and processed as immortal cell lines. Therefore, this platform could potentially be used for screening novel therapeutic agents that block cancer invasion and metastasis similar to clinical studies. The results demonstrated that this robust zebrafish xenotransplantation model provides an extremely encouraging solution for evaluating the proliferation potential of primary cells from biopsies or following surgery, a brilliant advantage for clinical studies. Furthermore, a variety of candidate MEK inhibitors can be screened for their ability to suppress pancreatic cancer by targeting multiple pathways (25). A typical screening using the larvae xenograft model can be completed under suitable time and space conditions (Fig. 5).

Taken together, our present study describes a zebrafish xenograft model that can be used to evaluate the inhibitory potential of KRAS pathway signaling inhibitors in human pancreatic cancer cell lines in vivo. This zebrafish model of xenotransplanted human pancreatic cancer cells showed a significant ability to perform tumor characteristic analysis in large cohorts of fish, allowing a robust statistical analysis in a short time frame ( $\sim 12$ days) and providing a rapid means of evaluating the effects of anticancer drugs in vivo. Based on this reliable model, new therapeutic drugs that block pancreatic cancer invasion and proliferation could potentially be developed by targeting the KRAS signaling pathway.

\section{Acknowledgements}

We acknowledge Professor H.Y. Song for her kind help in this study. The present study was supported in part by the National Natural Science Foundation of China (no. 81402582, no. 21202021) and by Shanghai Science and Technology Development Funds (no. 14YF1400600). 


\section{References}

1. Hidalgo M: Pancreatic cancer. N Engl J Med 362: 1605-1617, 2010.

2. Long J, Luo GP, Xiao ZW, Liu ZQ, Guo M, Liu L, Liu C, Xu J, Gao YT, Zheng Y, et al: Cancer statistics: Current diagnosis and treatment of pancreatic cancer in Shanghai, China. Cancer Lett 346: 273-277, 2014.

3. Siegel R, Ma J, Zou Z and Jemal A: Cancer statistics, 2014. CA Cancer J Clin 64: 9-29, 2014.

4. Jemal A, Bray F, Center MM, Ferlay J, Ward E and Forman D: Global cancer statistics. CA Cancer J Clin 61: 69-90, 2011.

5. Singh HM, Ungerechts G and Tsimberidou AM: Gene and cell therapy for pancreatic cancer. Expert Opin Biol Ther 15: 505-516, 2015 .

6. White R, Rose K and Zon L: Zebrafish cancer: The state of the art and the path forward. Nat Rev Cancer 13: 624-636, 2013.

7. Geiger GA, Fu W and Kao GD: Temozolomide-mediated radiosensitization of human glioma cells in a zebrafish embryonic system. Cancer Res 68: 3396-3404, 2008.

8. Berghmans S, Jette C, Langenau D, Hsu K, Stewart R, Look T and Kanki JP: Making waves in cancer research: New models in the zebrafish. Biotechniques 39: 227-237, 2005.

9. Zhang B, Shimada Y, Kuroyanagi J, Nishimura Y, Umemoto N, Nomoto T, Shintou T, Miyazaki T and Tanaka T: Zebrafish xenotransplantation model for cancer stem-like cell study and high-throughput screening of inhibitors. Tumour Biol 35: 11861-11869, 2014.

10. Jones S, Zhang X, Parsons DW, Lin JC, Leary RJ, Angenendt P, Mankoo P, Carter H, Kamiyama H, Jimeno A, et al: Core signaling pathways in human pancreatic cancers revealed by global genomic analyses. Science 321: 1801-1806, 2008.

11. Eser S, Schnieke A, Schneider G and Saur D: Oncogenic KRAS signalling in pancreatic cancer. Br J Cancer 111: 817-822, 2014.

12. Zorde Khvalevsky E, Gabai R, Rachmut IH, Horwitz E, Brunschwig Z, Orbach A, Shemi A, Golan T, Domb AJ, Yavin E, et al: Mutant KRAS is a druggable target for pancreatic cancer Proc Natl Acad Sci USA 110: 20723-20728, 2013.

13. Montagut $\mathrm{C}$ and Settleman J: Targeting the RAF-MEK-ERK pathway in cancer therapy. Cancer Lett 283: 125-134, 2009.

14. Frémin C and Meloche S: From basic research to clinical development of MEK1/2 inhibitors for cancer therapy. J Hematol Oncol 3: 8, 2010.

15. Davies SP, Reddy H, Caivano $M$ and Cohen P: Specificity and mechanism of action of some commonly used protein kinase inhibitors. Biochem J 351: 95-105, 2000.

16. Favata MF, Horiuchi KY, Manos EJ, Daulerio AJ, Stradley DA, Feeser WS, Van Dyk DE, Pitts WJ, Earl RA, Hobbs F, et al: Identification of a novel inhibitor of mitogen-activated protein kinase kinase. J Biol Chem 273: 18623-18632, 1998.
17. Kim ST, Lim H, Jang KT, Lim T, Lee J, Choi YL, Jang HL, Yi JH, Baek KK, Park SH, et al: Impact of KRAS mutations on clinical outcomes in pancreatic cancer patients treated with first-line gemcitabine-based chemotherapy. Mol Cancer Ther 10: 1993-1999, 2011.

18. Stoletov K, Montel V, Lester RD, Gonias SL and Klemke R: High-resolution imaging of the dynamic tumor cell vascular interface in transparent zebrafish. Proc Natl Acad Sci USA 104: 17406-17411, 2007

19. Gupta GP and Massagué J: Cancer metastasis: Building a framework. Cell 127: 679-695, 2006.

20. Morris JP IV, Wang SC and Hebrok M: KRAS, Hedgehog, Wnt and the twisted developmental biology of pancreatic ductal adenocarcinoma. Nat Rev Cancer 10: 683-695, 2010.

21. Wang X and Studzinski GP: Phosphorylation of raf-1 by kinase suppressor of ras is inhibited by 'MEK-specific' inhibitors PD 098059 and U0126 in differentiating HL60 cells. Exp Cell Res 268: 294-300, 2001.

22. Vaccaro V, Melisi D, Bria E, Cuppone F, Ciuffreda L, Pino MS, Gelibter A, Tortora G, Cognetti F and Milella M: Emerging pathways and future targets for the molecular therapy of pancreatic cancer. Expert Opin Ther Targets 15: 1183-1196, 2011.

23. McCubrey JA, Steelman LS, Chappell WH, Abrams SL, Wong EW, Chang F, Lehmann B, Terrian DM, Milella M, Tafuri A, et al: Roles of the Raf/MEK/ERK pathway in cell growth, malignant transformation and drug resistance. Biochim Biophys Acta 1773: 1263-1284, 2007.

24. Réjiba S, Wack S, Aprahamian M and Hajri A: K-ras oncogene silencing strategy reduces tumor growth and enhances gemcitabine chemotherapy efficacy for pancreatic cancer treatment. Cancer Sci 98: 1128-1136, 2007.

25. McCubrey JA, Steelman LS, Abrams SL, Chappell WH, Russo S, Ove R, Milella M, Tafuri A, Lunghi P, Bonati A, et al: Emerging MEK inhibitors. Expert Opin Emerg Drugs 15: 203-223, 2010

26. Gysin S, Lee SH, Dean NM and McMahon M: Pharmacologic inhibition of RAF $\rightarrow$ MEK $\rightarrow$ ERK signaling elicits pancreatic cancer cell cycle arrest through induced expression of $\mathrm{p} 27^{\mathrm{Kip} 1}$. Cancer Res 65: 4870-4880, 2005.

27. Bai X, Zhi X, Zhang Q, Liang F, Chen W, Liang C, Hu Q, Sun X, Zhuang $\mathrm{Z}$ and Liang $\mathrm{T}$ : Inhibition of protein phosphatase $2 \mathrm{~A}$ sensitizes pancreatic cancer to chemotherapy by increasing drug perfusion via HIF-1 $\alpha$-VEGF mediated angiogenesis. Cancer Lett 355: 281-287, 2014.

28. Ji F, Duan HG, Zheng CQ and Li J: Comparison of chloromethyl-dialkylcarbocyanine and green fluorescent protein for labeling human umbilical mesenchymal stem cells. Biotechnol Lett 37: 437-447, 2015. 